\title{
Intraventricular hemorrhage in premature infants Barbara Stonestreet
}

Address: Department of Pediatrics, Women \& Infants' Hospital of Rhode Island, 101 Dudley Street, Providence, RI 02905, USA

Email: Barbara Stonestreet - bstonestreet@wihri.org

from 52nd Annual Meeting of the Society for Research into Hydrocephalus and Spina Bifida

Providence, RI, USA. II-14 June 2008

Published: 3 February 2009

Cerebrospinal Fluid Research 2009, 6(SuppI I):S3 doi:I0.II86/1743-8454-6-SI-S3

This abstract is available from: http://www.cerebrospinalfluidresearch.com/content/6/SI/S3

(C) 2009 Stonestreet; licensee BioMed Central Ltd.

Dr. Stonestreet presented a case of an infant who was born 25 weeks gestation by spontaneous vaginal delivery with Apgar scores of $1^{1}$ and $5^{5}$. The infant was intubated because of decreased respiratory effort. He developed a pneumothorax on day 2 of life and bilateral grade 4 intraventricular hemorrhages. This case was used as a clinical example of infants that develop intraventricular hemorrhage. There is a large increase in brain maturation that occurs over the interval when infants develop intraventricular hemorrhage. From 23-32 weeks of gestation, important processes such as branching synaptagenesis, programmed cell death and reorganization of synapses are occurring in premature infants. The grading scale of LuAnn Papile has long been used to categorize grade of intraventricular hemorrhage. Fortunately, the incidence of intraventricular hemorrhage has decreased from the 1980's until 2006. In 2006, the incidence of all types of intraventricular hemorrhage were at 36 percent with severe types (Grades 3 and 4) representing 14 percent of infants $<1000 \mathrm{gm}$. Many clinical prenatal and postnatal factors predispose infants to intraventricular hemorrhage. Factors that can be protective against the neonatal development of intraventricular hemorrhage include maternal transport, optimal delivery, decreased use of ventilation where possible, limitation of intravenous volume boluses, limitation of the use of Dopamine, limitation of treatment with bicarbonate, avoiding pneumothorax and gentle handling. Hemorrhagic hydrocephalus occurs 1-3 weeks after intraventricular hemorrhage. In sixty-five percent of infants that develop post-hemorrhagic, it hydrocephalus resolves within 4 weeks. Fifteen to twenty percent of these infants require a shunt for post-hemorrhagic hydrocephalus. Serial lumbar punctures do not prevent the need for a shunt or improve neurologic outcomes. However, serial lumbar punctures and ventricular taps may be necessary as a temporizing effort. Futile therapies for post-hemorrhagic hydrocephalus include early lumbar punctures, the use of diuretics and fibrinolytic therapy to prevent shunt dependence. An intraventricular shunt may be necessary for infants with post-hemorrhagic hydrocephalus. However, the timing of the shunt placement is based largely upon clinical judgment and the size of the ventricles. When the ventricular dilation is $4 \mathrm{ml}$ higher than the $97^{\text {th }}$ percentile for ventricular width at a given, a shunt is most likely indicated. Recent data suggests that infants that require shunting for post-hemorrhagic hydrocephalus have poorer neurodevelopmental outcomes than infants with severe hydrocephalus that do not require a shunt. Nonetheless, at age 5 the infant that was presented by Dr. Stonestreet is doing quite well and is mainstreaming in a normal school environment. Early intervention therapies can help with the neurologic development even in infants that develop post-hemorrhagic hydrocephalus. 